\title{
Characterization of the Skin Mycobiome in Patients with Atopic Dermatitis
}

Song Hee Han, Ji Youn Hong, Jin Hee Kim, Joo Ran Hong, Hye In Cheon, Min Seok Hur, Byung Gon Choi, Yang Won Lee, Yong Beom Choe, Kyu Joong Ahn

Department of Dermatology, Konkuk University School of Medicine, Seoul, Korea

Background Patients with atopic dermatitis (AD) are known to be vulnerable to various infections of microbiome due to comprised skin barrier. Even though mycobiome, the skin fungal community, has key roles in immune-priming frontiers, studies referring to skin mycobiome in $A D$ are restrictive yet.

Objectives This study aimed to comparatively analyze the overall skin mycobiome between patients with $A D$ and healthy individuals in the Korean population. We also aimed to elucidate the role of Malassezia in pathophysiology of AD.

Methods Skin swab samples from the antecubital fossae of 8 patients with $A D$ and 8 healthy controls were analyzed. Using sequencing method followed by direct DNA extraction and molecular PCR, taxonomic compositions of fungi at stepwise level ranks were analyzed. The phylogenic marker used was internal transcribed spacer 2 regions of DNA.

Results According to diversity analyses using Shannon diversity index (SDI), patients with $A D$ were shown to have more diverse fungi than healthy controls at genus and species level.

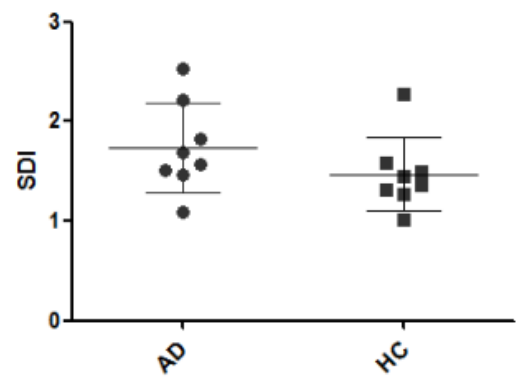

(A) Phylum

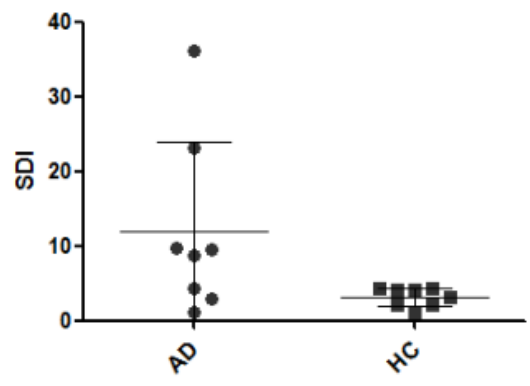

(B) Genus

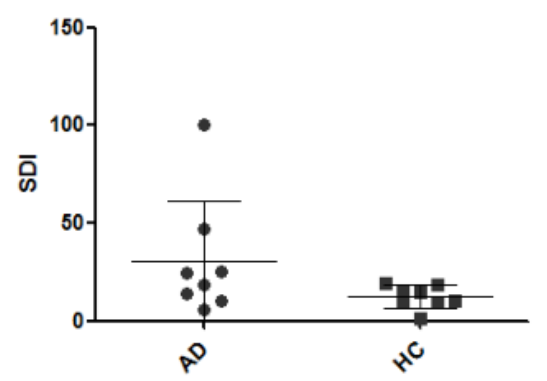

(C) Species
Fig. 1. Diversity analyses using SDI at phylum (A), genus (B), and species (C) level

Circular dots and square dots represent SDIs of samples from $A D$ and $H C$, respectively. $A D$, atopic dermatitis; $H C$, control

Malassezia globosa and Malassezia restricta were prevalent in all samples across both study groups. However, Malassezia slooffiae, Malassezia obtusa and Malassezia yamatoensis were identified only in the samples from AD group, while Malassezia furfur was detected more frequently in the healthy controls.

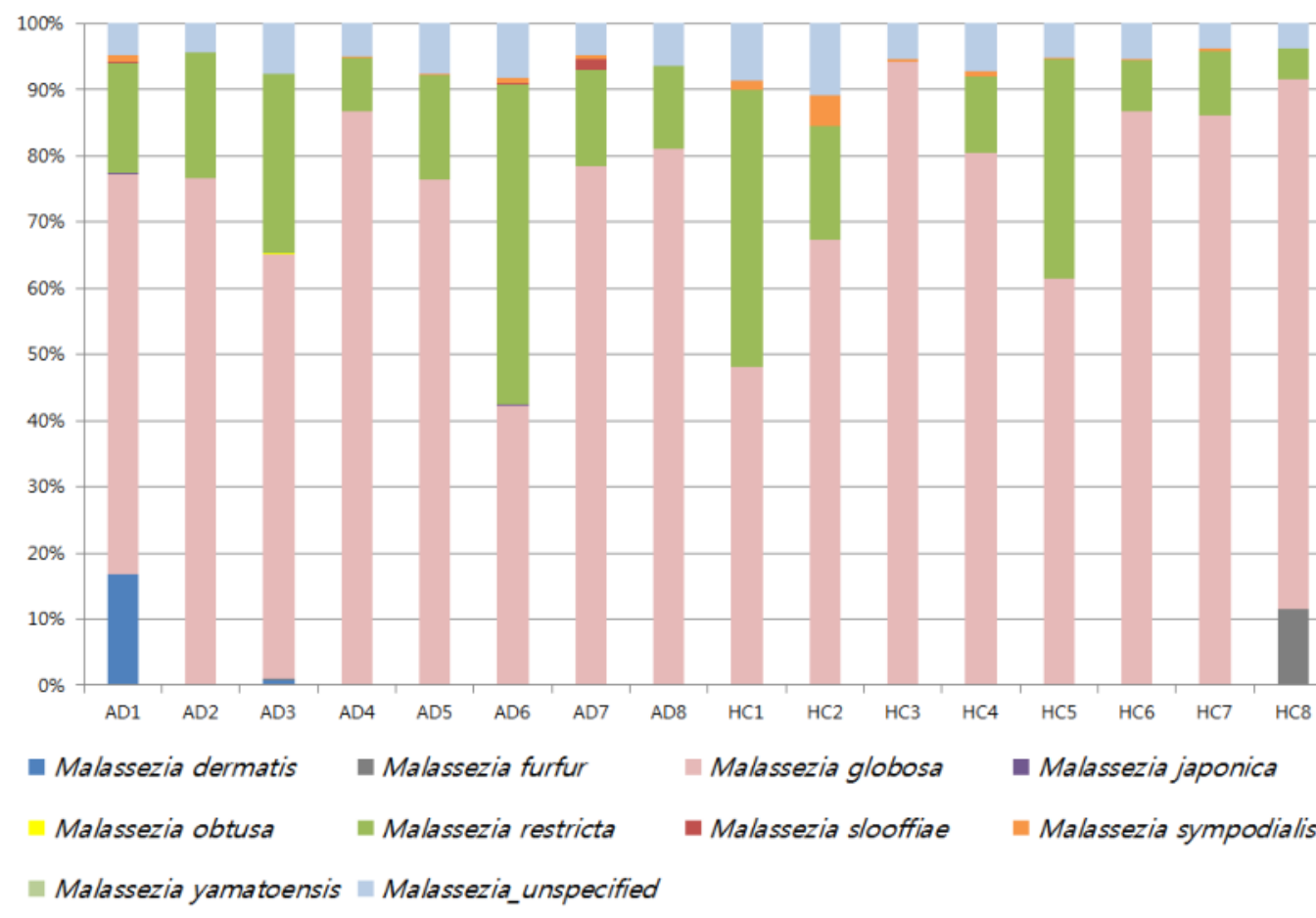

Fig. 2. Relative abundance of Malassezia species

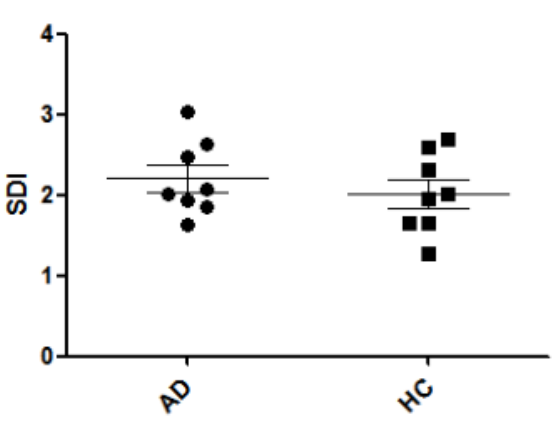

Fig. 3. Diversity analysis on Malassezia species using SDI
The difference of mean SDIs of the Malassezia components between 2 groups was not significant, in contrast that the difference existed in terms of overall fungal species level.

Conclusion We detected a tendency of higher intraand inter-personal skin fungal diversities in $A D$ populations compared with healthy populations. Simultaneously, non-Malassezia diversity was more noticeable in $A D$ populations. Although this study was limited by the small sample size, it would be a preliminary study, contributing to set up the database of skin mycobiome in patients with $A D$.

\section{References}

1. Cui L, Morris A, Ghedin E. The human mycobiome in health and disease. Genome Med 2013;5:63.

2. Prohic A, Jovovic Sadikovic T, Krupalija-Fazlic M, Kuskunovic-Vlahovljak S. Malassezia species in healthy skin and in dermatological conditions. Int $\mathrm{J}$ Dermatol 2016;55:494-504.

3. Pereira SG, Moura J, Carvalho E, Empadinhas N. Microbiota of Chronic Diabetic Wounds: Ecology, Impact, and Potential for Innovative Treatment Strategies. Front Microbiol 2017;8:1791.

4. Glatz M, Bosshard PP, Hoetzenecker W, SchmidGrendelmeier P. The Role of Malassezia spp. in Atopic Dermatitis. J Clin Med 2015;4:1217-1228. 